\title{
Obstrucción del tracto de salida de la unión esofagogástrica: ¿Qué es y cómo manejarla?
}

\author{
Andrés F. Ardila-Hani, Ana María Leguízamo, Valeria Costa y Albis C. Hani de Ardila*
}

Unidad de Gastroenterología, Hospital Universitario San Ignacio, Pontificia Universidad Javeriana, Bogotá, Colombia

\section{RESUMEN}

Los trastornos motores del esófago se categorizan mediante la Clasificación de Chicago 3.0 (CC) en trastornos mayores y menores de la peristalsis; y aquellos con obstrucción del tracto de salida, subdivididos en acalasia y obstrucción del tracto de salida de la unión esofagogástrica (OTSUEG). El presente artículo tiene como objetivo revisar los tipos de OTSUEG, su etiología, manifestaciones clínicas, fisiopatología subyacente, las distintas pruebas diagnósticas para su estudio y el abanico de opciones terapéuticas. La OTSUEG comprende un grupo heterogéneo de pacientes que se caracteriza por hallazgos manométricos de alteración en la unión esofagogástrica (UEG), con presencia de peristalsis intacta o débil del cuerpo esofágico, de tal forma que no se cumplan los criterios para acalasia. Puede ser causada no solo por patología funcional (idiopática) sino estructural (secundaria). La disfagia es el síntoma más común, seguida por dolor torácico, regurgitación y pirosis. El hallazgo de OTSUEG en ausencia de etiología secundaria se acompaña de una incertidumbre clínica. Algunos parecen tener un estadio temprano de acalasia, mientras otros no presentan casi ningún síntoma obstructivo y el hallazgo de integrada de la presión de relajación (IPR) elevada puede ser solo un hallazgo incidental sin implicación o relevancia clínica. El diferenciar la OTSUEG secundaria es importante, debido a que estos pacientes requerirán un tratamiento distinto a aquellos pacientes con OTSUEG primaria o idiopática en muchas ocasiones. Dentro del armamentario diagnóstico se encuentran la endoscopia, el esofagograma y la manometría de alta resolución (MAR) para identificar causas subyacentes de relajación incompleta de la UEG. La ecoendoscopia y la tomografía computarizada (TC) nos pueden ayudar a excluir patología infiltrativa o inflamatoria. Aunque no existen tratamientos curativos para los trastornos de la OTSUEG, existen 
opciones de tratamiento médico, endoscópico y quirúrgico. Se recomienda un tratamiento individualizado de esta condición, teniendo en cuenta factores como los síntomas del paciente y la severidad de estos, y el tipo de OTSUEG (funcional vs. secundaria), entre otras cosas, recordando que en un porcentaje importante de personas puede haber resolución espontánea de los síntomas durante el seguimiento. (NeuroGastroLatam Rev. 2017;2:64-77) Corresponding author: Albis C. Hani de Ardila, albishani@gmail.com

Palabras clave: Obstrucción del tracto de salida. Unión esofagogástrica. Trastornos motores funcionales esofágicos.

\section{ABSTRACT}

The motor esophageal disorders are categorized according to the Chicago Classification 3.0 into major and minor disorders of peristalsis; and those with outflow obstruction; divided in achalasia and esophagogastric junction outflow obstruction (EGJOO). The present study has as an objective to revise the types of EGJOO, their etiology, clinical manifestations, pathophysiology, diagnostic tests and different treatment options. EGJOO comprises a heterogeneous group of patients characterized by manometric findings of an alteration in the EGJ, with the presence of intact or weak peristalsis of the esophageal body, in such a way that the criteria for achalasia are not met. It can be caused not only by functional pathology (idiopathic) but structural (secondary) pathology. Dysphagia is the most common symptom, followed by chest pain, regurgitation and heartburn. The finding of EGJOO in the absence of secondary etiology is accompanied by clinical uncertainty. Some seem to have an early stage of achalasia, while others have almost no obstructive symptoms and the finding of a high integrated relaxation pressure may be only an incidental finding without clinical implication or relevance. Differentiating secondary EGJOO is important because these patients will require a different management on many occasions compared to patients with primary or idiopathic EGJOO. Within the diagnostic armamentarium, we count with endoscopy, esophagogram and high-resolution manometry to identify underlying causes of incomplete relaxation of the EGJ. Endoscopic ultrasound and computed tomography can help us exclude infiltrative or inflammatory pathology. Although there are no curative treatments for EGJOO disorders, there are options for medical, endoscopic and surgical management. Individualized management of this condition is recommended, taking into account factors such as the symptoms of the patient and the severity of these symptoms, and the type of EGJOO (functional vs. structural), among others, remembering that in a significant percentage of patients there might be a spontaneous resolution of symptoms during follow-up.

Key words: Outflow obstruction. Esophagogastric junction. Esophageal functional motor disorders 


\section{INTRODUCCIÓN}

La UEG es un punto crucial en la fisiopatología esofágica, que cumple un papel en condiciones como la enfermedad por reflujo gastroesofágico (ERGE) o los trastornos motores funcionales del esófago ${ }^{1}$. Por este motivo la MAR tiene un rol central en la evaluación de la competencia (función de barrera) y relajación de la UEG.

La evaluación de la UEG tiene varias dificultades, ya que es un esfínter complejo compuesto por el diafragma crural (DC) y el esfínter esofágico inferior (EEI). La presión de la UEG va a variar según la respiración, el tiempo y la deglución; como también su morfología, la cual analizada desde el punto de vista manométrico ha sido caracterizada en tres subtipos': tipo 1, en la que hay una superposición del EEI y el DC; tipo 2, con una separación axial del EEI y el DC $<2 \mathrm{~cm}$, y la tipo 3, con una separación $\geq 2 \mathrm{~cm}$ entre el EEI y el DC. La tipo 3, a su vez, se subdivide en 3a, cuando la IPR permanece a nivel del DC, $y$ en $3 b$, cuando la IPR está a nivel del EEI. Esto se evidencia en la manometría por una disminución de la presión del EEI durante la inspiración en la tipo 3a y un aumento en la presión del EEI con la inspiración en la 3b.

Debido a la existencia de numerosos factores que influyen en la relajación de la $\mathrm{UEG}^{3}$ se desarrolló una medida de presión topográfica esofágica llamada PIR ${ }^{4}$. Esta variable está ubicada en el lugar espacial necesario para capturar el movimiento axial del EEI en el momento posdeglutorio, cuando el esfínter esofágico superior se relaja y hasta que finaliza la contracción peristáltica distal (o 10 segundos en ausencia de peristalsis). El valor reportado será el promedio de los 4 segundos de máxima relajación luego de la deglución, en un total de 10 degluciones, con un valor final que será la mediana de todas las degluciones, siendo $15 \mathrm{mmHg}$ el límite superior de lo normal.

El desarrollo de la CC permitió un método para interpretar los estudios de MAR y clasificar los trastornos motores esofágicos. La última versión de la CC es la v3.0, publicada en el año $2015^{2}$, la cual permitió categorizar los trastornos motores esofágicos en mayores, entre los que están los trastornos de motilidad distintos a la acalasia y la OTSUEG, que no se encuentran presentes en individuos sanos; y trastornos menores, en los cuales existen alteraciones en el tránsito del bolo esofágico con base en los patrones de vigory contractilidad, donde se incluyen la motilidad esofágica inefectiva y la peristalsis fragmentada. Se ha encontrado alguna superposición de estos trastornos menores en individuos sanos y siguen siendo un motivo de debate incluso en la CC v3.0.

Luego de definir la relajación de la UEG, el siguiente paso en el análisis de la MAR es caracterizar la peristalsis. Para esto se evalúa la integral de contractilidad distal (ICD) y la latencia distal (LD). El primero evalúa la longitud, el vigor y la persistencia de la contracción esofágica distal desde el punto de mínima presión en la zona de transición, la cual separa los dos primeros segmentos contráctiles esofágicos, hasta el punto de presión mínima que separa los otros dos segmentos, los cuales se alojan en el músculo liso esofágico del EEI ${ }^{5}$. El ICD va a ser calculado con la multiplicación de la amplitud promedio de la contracción en el esófago distal $(\mathrm{mmHg})$, la 

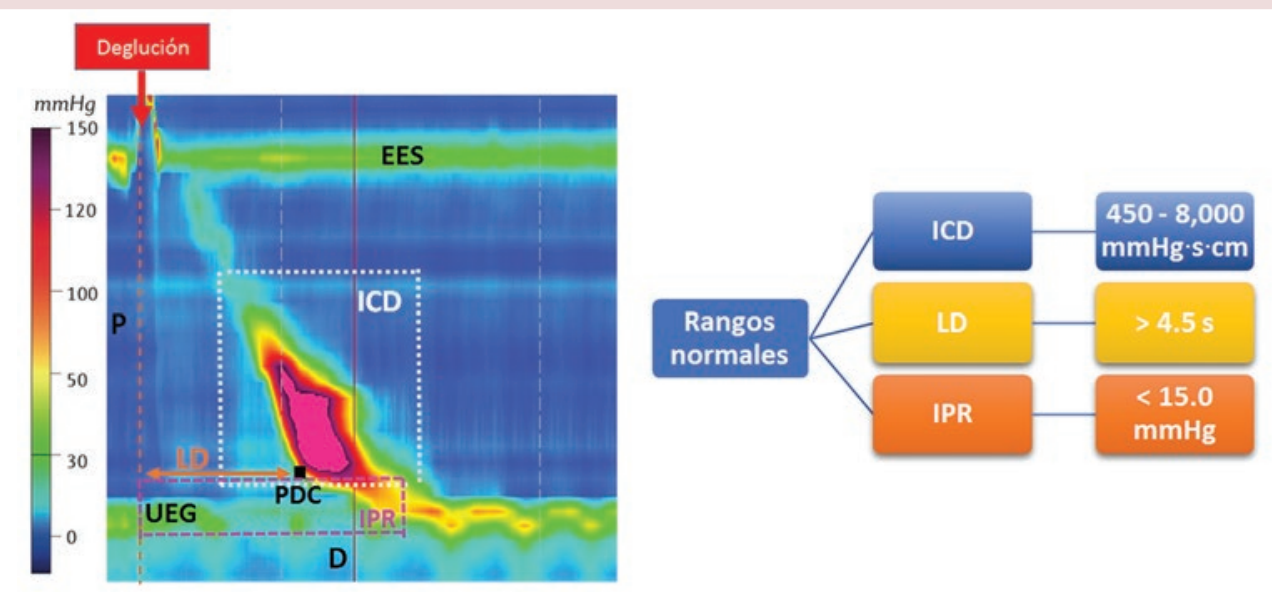

Figura 1. Parámetros manométricos topográficos para describir la peristalsis según la CC 3.0.

EES: esfínter esofágico superior; UEG: unión esofagogástrica; P: valor de presión más baja o zona de transición, que separa los dos primeros segmentos contráctiles; D: la mínima presión que separa los otros dos segmentos contráctiles; ICD: integral de la contractilidad distal, que consiste en la sumatoria de la amplitud contráctil en el rango entre el punto $\mathrm{P}$ y el $\mathrm{D}$, y se describe en unidades de mmHgcm; PDC: punto de desaceleración contráctil, localizado en el esófago distal, nos indica la transición desde la peristalsis esofágica hasta la fase tardía del vaciamiento esofágico. LD: latencia distal, la cual se mide desde el punto de relajación del EES (demarcado por la línea vertical intermitente naranja) hasta el CDP; IPR: integrada de la presión de relajación, mide la relajación de la UEG, calculada en una ventana de 10 s comenzando con la relajación del EES; CC: clasificación de Chicago.

duración de la contracción (segundos) y la longitud del segmento esofágico distal $(\mathrm{cm})$. Es, por lo tanto, expresada en $\mathrm{mmHg} \cdot \mathrm{s} \cdot \mathrm{cm}^{6}$.

La LD permite evaluar la presencia de contracciones prematuras, indicando una alteración en las neuronas inhibitorias del plexo mientérico esofágico. Se mide desde la relajación del esfínter esofágico superior hasta el punto de desaceleración contráctil (PDC), que es el punto de inflexión justo proximal a la UEG que demarca la transición entre la peristalsis hasta la fase tardía del vaciamiento esofágico. Una $\mathrm{LD}<4.5$ segundos define una contracción prematura (Fig. 1).

Los trastornos que cursan con PIR elevado

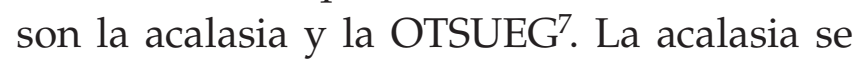
subdivide así mismo en tres subtipos manométricos de contractilidad: la tipo I, con ausencia de presurización en el esófago; la tipo II, con presurización panesofágica y la tipo III, con contracciones prematuras (espásticas) en $\geq 20 \%{ }^{4}$. La etiología de esta condición no está clara y su diagnóstico casi siempre se basa en los hallazgos de la MAR. Sin embargo, existen casos en los que podemos encontrar en la acalasia una integrada de la presión de relajación (IPR) $<15 \mathrm{mmHg}$ o incluso una peristalsis preservada. Esto puede deberse a que el curso de la enfermedad tiene rangos de tiempo variables en donde puede haber una transición gradual entre la función normal y la ausencia de peristalsis con OTSUEG, con puntos intermedios en la historia natural de la acalasia en los que estas anormalidades no cumplen los umbrales diagnósticos manométricos ${ }^{8}$.

La OTSUEG es una condición caracterizada por hallazgos manométricos de alteración en la función de la UEG (IPR elevada), pero, al 


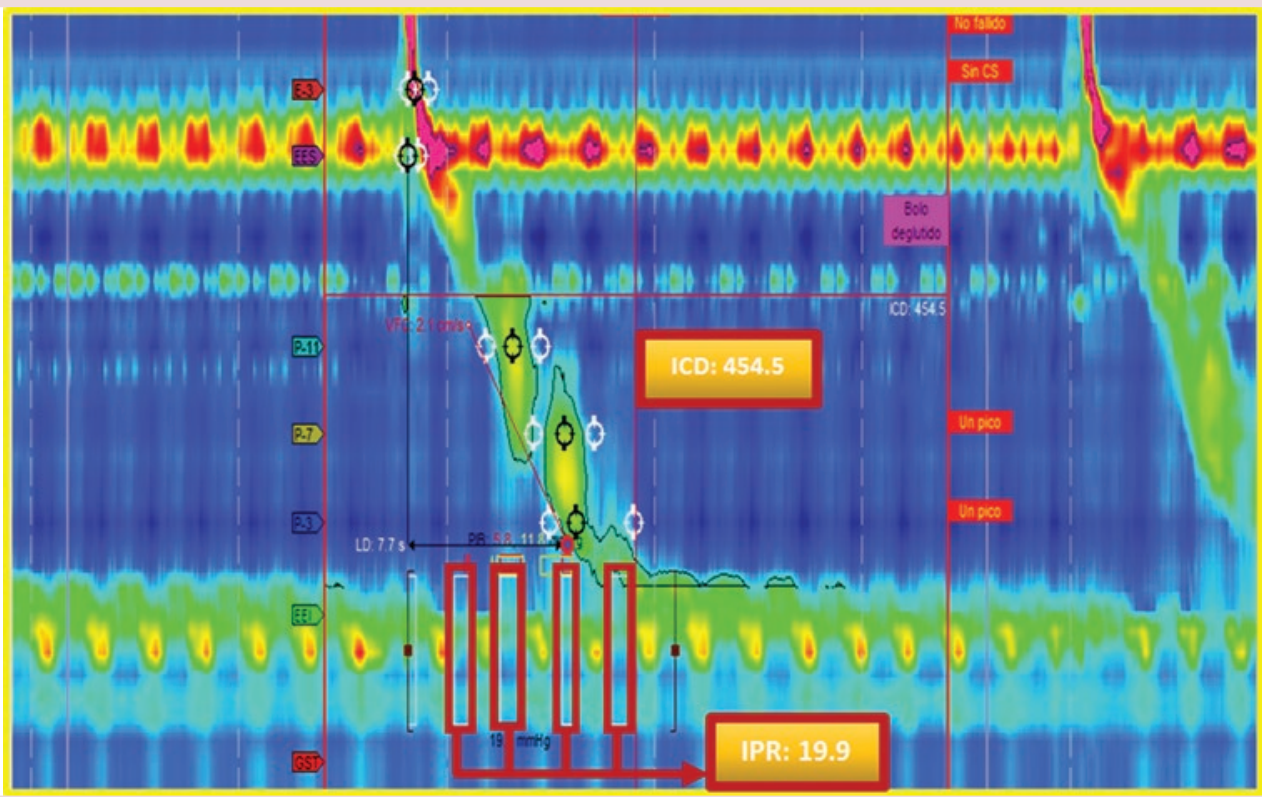

Figura 2. MAR consistente con OTSUEG. Se evidencia una alteración en la función de la UEG (IPR elevada) posterior a la deglución, pero característicamente hay una peristalsis que puede ser intacta o débil del cuerpo esofágico (intacta en este caso), de tal forma que no cumple con los criterios para acalasia.

UEG: unión esofagogástrica; IPR: integrada de la presión de relajación; MAR: manometría de alta resolución; OTSUEG: obstrucción del tracto de salida de la unión esofagogástrica.

menos en cierto porcentaje existe peristalsis intacta o débil del cuerpo esofágico, de tal forma que no se cumplen los criterios para acalasia $^{9}$ (Fig. 2). Se ha referido en numerosas ocasiones a esta condición como una variante de acalasia; sin embargo, puede ser causada no solo por patología funcional, sino también estructural. Este artículo de revisión tiene como objetivo describir los tipos de OTSUEG, su etiología, manifestaciones clínicas, fisiopatología subyacente, las distintas pruebas diagnósticas para su estudio y el abanico de opciones terapéuticas. Para esto se realizó una revisión narrativa de la literatura de esta condición, que ha sido descrita recientemente y cuyo diagnóstico se hace cada vez más común debido al uso creciente de la MAR en la práctica clínica.

\section{ETIOLOGÍA}

La OTSUEG tiene una significancia clínica incierta ${ }^{10}$. Poco se conoce sobre su incidencia, etiopatogenia, evolución y tratamiento más apropiado.

Comprende un grupo heterogéneo de pacientes, ¿es acaso una variante de la acalasia con una expresión temprana o incompleta (OTSUEG funcional o idiopática) ${ }^{11}$ o será resultado de procesos mecánicos o estructurales no detectados? (hernia hiatal, estenosis esofágica péptica o posquirúrgica, esofagitis eosinofílica, anillo de Schatzki, compresión extrínseca, hernia paraesofágica, compresión vascular, banda gástrica o divertículo epifrénico, entre otros) $)^{12}$ (Tabla 1). 
TABLA 1. Causas secundarias de OTSUEG

\begin{tabular}{|l|l|}
\hline \multicolumn{2}{|l|}{ Causas secundarias de OTSUEG } \\
\hline Hernia hiatal & Banda gástrica \\
\hline $\begin{array}{l}\text { Estenosis esofágica } \\
\text { (péptica o posquirúrgica) }\end{array}$ & Divertículo epifrénico \\
\hline Esofagitis eosinofílica & Hernia paraesofágica \\
\hline Anillo de Schatzki & Compresión vascular \\
\hline Compresión extrínseca & Tumores \\
\hline Uso de opioides & \\
\hline
\end{tabular}

¿Podrá ser la OTSUEG una variante normal en los casos leves, siendo en esos casos un artefacto del estudio manométrico?

Asimismo se ha descrito que el uso de opiáceos puede estar asociado a un aumento en la amplitud peristáltica y una relajación incompleta del EEI ${ }^{13}$. Esto puede tener implicaciones terapéuticas, ya que descontinuar el tratamiento podría mejorar la disfunción manométrica esofágica, y esto es algo a tener en cuenta en los pacientes con OTSUEG ${ }^{14}$.

\section{PRESENTACIÓN CLÍNICA}

La presentación clínica de la OTSUEG idiopática parece ser heterogénea. La disfagia es el síntoma más común, afectando aproximadamente a un $40 \%$ de las personas en las cohortes más grandes, seguido por el dolor toráciCo, la regurgitación y la pirosis ${ }^{15,16}$.

Aunque no son muchos los casos descritos en la literatura, sí existe en ocasiones progresión de la OTSUEG hacia acalasia. Lynch, et al. describieron dos pacientes que evolucionaron a acalasia tipo II, diagnosticándose esta a los 16 y 22 meses de seguimiento ${ }^{16}$. Generalmente estos son pacientes con persistencia de los síntomas (disfagia) en el tiempo. Otros estudios han reportado casos similares. Es el caso de Clayton, et al., en cuyo estudio dos de 15 pacientes que presentaban disfagia para sólidos fueron tratados con inyecciones de toxina botulínica con mejoría sintomática transitoria, pero al seguimiento a 12-24 meses comenzaron a presentar disfagia para sólidos y líquidos, con una MAR consistente con acalasia tipo III $^{11}$.

Van Hoeij, et al. ${ }^{10}$ reportaron ocho pacientes con síntomas típicos de acalasia (disfagia) y que recibieron tratamiento con inyecciones de toxina botulínica o DN. Durante el seguimiento, tres de estos pacientes fueron diagnosticados con acalasia en una nueva MAR, a los 11 y 18 meses.

Series de casos de OTSUEG han encontrado también que varios pacientes con esta condición son mínimamente sintomáticos o asintomáticos ${ }^{10}$.

\section{DIAGNÓSTICO}

El diferenciar la OTSUEG secundaria es importante, debido a que estos pacientes requerirán un tratamiento distinto a aquellos pacientes con OTSUEG EGJOO primaria o idiopática en muchas ocasiones.

Existen varias opciones diagnósticas en estos pacientes además de la endoscopia y la MAR, en busca de identificar causas subyacentes para la relajación incompleta de la UEG. Están la TC y la ecoendoscopia para excluir patología infiltrativa o inflamatoria ${ }^{17}$.

El hallazgo de una OTSUEG en ausencia de etiología secundaria se acompaña de una 
incertidumbre clínica. Algunos parecen tener un estadio temprano de acalasia, mientras otros no presentan casi ningún síntoma obstructivo y el hallazgo de una IPR elevada puede ser solo un hallazgo incidental sin implicación o relevancia clínica.

\section{Esofagograma}

El esofagograma es una prueba de bajo costo con bajas tasas de radiación que con el tiempo se ha convertido en parte del armamentario diagnóstico en todos los pacientes con disfagia esofágica. Provee información detallada de la anatomía esofágica y también del vaciamiento de líquidos y de sólidos (tableta). Esta última prueba, para valorar la deglución de sólidos, utiliza una tableta de bario de $13 \mathrm{~mm}$, siendo anormal el examen cuando la tableta es retenida luego de 5 minutos de haberse ingerido. Aunque el papel del esofagograma en los pacientes con acalasia está bien establecido, existe muy poca literatura sobre el rol de la tableta de bario en los pacientes con acalasia no tratada, OTSUEG o disfagia sin presencia de acalasia.

El esofagograma se usa para evaluar el vaciamiento esofágico en pacientes con acalasia antes y después del tratamiento. La mejoría de los síntomas y el vaciamiento esofágico postratamiento ha mostrado ser predictor de remisión sintomática a largo plazo, mientras que la mejoría únicamente de los síntomas y no del vaciamiento ha sido predictor de la recurrencia sintomática durante el seguimiento. No se sabe mucho sobre la precisión predictiva de este examen en pacientes con acalasia no tratada $y$ en pacientes con OTSUEG.
Un estudio recientemente publicado buscó evaluar el tránsito del bolo por esofagograma en pacientes con acalasia (tipos I, II y III), disfagia sin presencia de acalasia y OTSUEG, y determinar la capacidad de este examen para diferenciar estos grupos de pacientes ${ }^{18}$. Generalmente, el aclaramiento para líquidos se considera normal si la altura de la columna de bario es $<5 \mathrm{~cm}$ en el esófago distal luego de 5 minutos $^{19,20}$.

Al comparar todos los tipos de acalasia con el grupo de OTSUEG, se vio que todas las acalasias tuvieron una mayor altura promedio de la columna de bario al minuto y a los $5 \mathrm{mi}-$ nutos. Así mismo, el ancho de la columna de bario fue significativamente mayor en la acalasia I y II en relación al grupo con OTSUEG. La retención de la tableta de bario de $13 \mathrm{~mm}$ fue significativamente mayor en todos los subtipos de acalasia en comparación con los pacientes con OTSUEG.

No se observaron diferencias significativas en la altura ni en el ancho de la columna de bario entre los pacientes con OTSUEG y aquellos sin acalasia. Basándose en esta pobre o fallida precisión del esofagograma para separar los grupos de pacientes con OTSUEG de los que tienen disfagia sin presencia de acalasia, el estudio recomienda no utilizar el esofagograma como una prueba sustituta de la MAR, sino más bien como una prueba complementaria.

Un estudio mostró la presencia de anormalidades en el $72 \%$ de los esofagogramas realizados en 40 pacientes con OTSUEG ${ }^{21}$. La anormalidad más frecuente fue la presencia de hernia hiatal en 20 pacientes, seguida por espasmo o dismotilidad (17 pacientes) y 
disminución del calibre en la UEG (10 pacientes). En cinco pacientes se identificó un anillo de Schatzki y menos frecuentemente se describió compresión extrínseca de la UEG, malrotación y vólvulo gástrico.

\section{Endoscopia de vías digestivas altas}

La endoscopia puede ayudar a identificar causas secundarias de OTSUEG. En estudios con poblaciones de pacientes con OTSUEG se describen endoscopias de vías digestivas altas (EVDA) normales en aproximadamente el 46\% de los pacientes ${ }^{21}$. Hallazgos comúnmente descritos son la presencia de hernia hiatal, y menos frecuentemente anillo de Schatzki, esofagitis (erosiva o eosinofílica), candidiasis y masa a nivel de la UEG. Van Hoeij, et al. identificaron en 12 de 47 pacientes con OTSUEG una alteración mecánica con la EVDA, entre ellas estómago intratorácico, bandas gástricas, $\mathrm{CA}$ esofágico, metástasis esofágicas, compresión vascular, anillo de Schatzki y fundoplicatura ${ }^{16}$.

\section{Manometría de alta resolución - impedanciometría}

El diagnóstico va a estar definido por una elevación de la IPR en conjunto con una peristalsis preservada o débil, de tal forma que no cumpla los criterios para acalasia. En varias ocasiones esto puede estar acompañado por una presión intrabolo (PIB) elevada, que se considera una consecuencia lógica de la alteración en la relajación de la $\mathrm{UEG}^{17}$. Sin embargo, se ha descrito que no hay diferencias significativas en los síntomas, parámetros de MAR o efecto en el tratamiento entre pacientes con y sin elevación de la PIB $^{16}$.
La MAR con impedancia intraluminal ofrece una oportunidad para caracterizar mejor la importancia funcional de la OTSUEG idiopática.

El tránsito completo del bolo (TCB) está dado por una entrada del bolo en el sitio de registro de la impedancia más proximal y la salida subsecuente en los tres puntos distales de registro, indicado por una caída apropiada y retorno a la impedancia basal. En la mayoría de sujetos sanos el TCB es normal en $\geq 8$ de 10 degluciones líquidas ${ }^{22,23}$. Existen estudios que han mostrado un aclaramiento pobre del bolo en pacientes con OTSUEG, describiéndose además que el compromiso del aclaramiento es menos severo que en pacientes con acala$\mathrm{sia}^{24}$. Asimismo se ha visto que el grado de elevación de la IPR no está asociado necesariamente con el aclaramiento del bolo.

Diversos estudios han evaluado los parámetros manométricos en pacientes con OTSUEG con persistencia de los síntomas en comparación con los que presentaron resolución de los síntomas, encontrando diferencias significativas para variables como el ICD e IPR máxima, siendo estas mayores en los pacientes con persistencia de los síntomas ${ }^{25}$. Este mismo estudio mostró que tanto la PIB como el aclaramiento del bolo no difirió entre pacientes con OTSUEG sintomáticos de aquellos con resolución de los síntomas. Sin embargo, al comparar las variables manométricas entre pacientes con OTSUEG con un grupo control de voluntarios sanos, Pérez-Fernández, et al. describieron valores más elevados de PIB, IPR y una mayor proporción de tránsito esofágico incompleto en los pacientes con OTSUEG ${ }^{15}$.

Otro hallazgo manométrico reportado en pacientes con OTSUEG es la variabilidad del 
ICD, con casos de hipocontractilidad e hipercontractilidad. Se describe la asociación de esófago hipercontráctil (jackhammer) con $\mathrm{OTSUEG}^{15}$. Roman y Kharilas describieron que el espasmo esofágico distal y el esófago hipercontráctil podían estar asociados con OTSUEG ${ }^{26}$. Gyawali, et al. observaron también que los pacientes con OTSUEG presentaban un patrón motor caracterizado por contracciones con múltiples picos, una amplitud esofágica distal alta y una duración de contracción prolongada ${ }^{27}$.

Dada esta asociación de la OTSUEG y la hipercontractilidad, algunos autores han sugerido que los trastornos esofágicos espásticos pueden progresar a acalasia ${ }^{28}$. En estos pacientes en los que se observa progresión, la vigilancia clínica deberá ser más rigurosa, sin embargo, no existen tiempos definidos para la realización de exámenes de seguimiento como MAR, esofagogramas o ecoendoscopias. El tiempo de realización de estos estudios deberá determinarse de manera individualizada.

Recientemente, un estudio describió que los hallazgos en la MAR realizada únicamente con diez degluciones líquidas pueden fallar en llegar a un diagnóstico definitivo que explique los síntomas del paciente ${ }^{29}$. Este estudio mostró que la inclusión de un protocolo de MAR con degluciones sólidas aumenta el rendimiento diagnóstico de los trastornos motores esofágicos. Este rendimiento fue mayor en pacientes con disfagia, sin embargo, también fue visto en pacientes referidos por síntomas de reflujo. Se observó que el trastorno que más comúnmente fue detectado por la manometría con degluciones sólidas y no por la manometría con degluciones líquidas fue la OTSUEG. La relevancia clínica de este hallazgo, además, radica en que cerca del 90\% de los pacientes con OTSUEG tuvieron síntomas con la presurización esofágica. Hallazgos similares de un mayor rendimiento diagnóstico de la OTSUEG con líquidos viscosos han sido descritos por Xiao, et al. ${ }^{30}$

A pesar de los resultados descritos, en donde se evidencia una ventaja aparente de las degluciones sólidas respecto a las líquidas, antes de establecer cualquier conclusión, hace falta todavía una estandarización del protocolo de análisis de la comida sólida y del esquema diagnóstico de esta prueba, ya que no es igual a la establecida por la CC v3.0.

\section{Ecoendoscopia}

Aunque la MAR nos aporta una información fisiológica detallada y un mapa topográfico del esófago basado en presiones, hay que reconocer que puede estar cegada para identificar anomalías de presión por alteraciones anatómicas. Por esto se hace necesaria en ocasiones la realización de estudios imagenológicos, siendo uno de estos la ultrasonografía endoscópica (USE).

En pacientes con acalasia y estados hipercontráctiles se ha descrito un engrosamiento marcado de la muscular propia en comparación con los controles. Asimismo se observó en varios de estos pacientes una hipertrofia tanto de las capas musculares circular y longitudinal, con una mayor área total transversal de músculo en comparación a los controles ${ }^{31}$.

La USE nos permite evaluar la presencia de malignidad, nódulos linfáticos, compresiones vasculares o enfermedad infiltrativa. 
Un estudio de Krishanan, et al. ${ }^{32}$ evaluó el papel de la USE en pacientes con trastornos motores esofágicos. La principal indicación de la USE fue evaluar pseudoacalasia o compresión extrínseca de la UEG. Dentro del grupo de pacientes con IPR $\geq 15 \mathrm{mmHg}$ (acalasia y OTSUEG EGJOO), aquellos con OTSUEG tuvieron el mayor espesor de la pared esofágica, de la muscular propia y del EEI. En nueve pacientes (15\%) se encontraron lesiones significativas con la USE, de estos, cuatro tenían acalasia, dos una compresión aórtica, otro una masa intramural y el último un leiomioma, Cinco pacientes tenían OTSUEG, documentándose en uno de ellos una masa intramural, los otros con un anillo muscular congénito, compresión aórtica, sarcoidosis y en otro ausencia de células ganglionares, con inflamación perineural en la biopsia, confirmándose finalmente un diagnóstico de acalasia.

La USE puede entonces modificar el tratamiento de los pacientes en ciertos casos, por lo que puede ser útil para estudiar con más detalle los pacientes con OTSUEG con una potencial etiología mecánica o anatómica. Por otro lado, hay estudios en los que la mayoría de los pacientes llevados a USE tuvieron resultados normales y en aquellos con anormalidades, estas ya habían sido identificadas por otros métodos (esofagograma, EVDA), no viéndose modificado el tratamiento médico tras los hallazgos endosonográficos ${ }^{21}$.

En cuanto a la determinación del espesor de la pared esofágica, estudios previos describen hallazgos variables, principalmente aquellos que evalúan pacientes con acalasia $^{31}$. Esta variabilidad puede deberse a diferencias en la técnica de USE, contracción esofágica durante la medición, tortuosidad del esófago y dilatación esofágica, entre otras.

\section{EndoFlip}

Este dispositivo consiste en un largo catéter que está unido a una bolsa cilíndrica que contiene varios electrodos unidos estrechamente, a través de los cuales se genera una corriente. Los valores de impedancia obtenidos permiten un cálculo del área luminal transversal (planimetría de impedancia). Al computar esta área luminal transversal y evaluar su relación con la presión en la bolsa, el EndoFlip permite cuantificar la distensibilidad de la UEG y genera un esquema tridimensional de un órgano distensible $^{33}$. La mayoría de los estudios con este equipo han sido realizados en pacientes con acalasia, en los cuales se ha descrito que generalmente la distensibilidad de la UEG se encuentra significativamente reducida en comparación a controles sanos ${ }^{34}$. Se ha descrito también que en pacientes con acalasia la distensibilidad de la UEG aumenta con la terapia y es mejor predictor de los síntomas o la recurrencia de estos que la presión del $\mathrm{EEI}^{35}$. Por estos motivos se ha encontrado un potencial en el EndoFlip para la valoración clínica de estos pacientes.

El EndoFlip se hace útil como parte de las herramientas disponibles para el estudio de la disfagia; los hallazgos encontrados pueden determinar si se requieren estudios adicionales o algún tipo de terapia, por ejemplo, en casos con OTSUEG. 


\section{TRATAMIENTO}

La OTSUEG es un trastorno que involucra un grupo heterogéneo de pacientes, de los cuales solo algunos se han beneficiado de las terapias utilizadas comúnmente para la acalasia ${ }^{36}$. Por este motivo estos pacientes requieren siempre de una evaluación clínica más intensa (por ejemplo, TC, USE) para encontrar la etiología.

Todos los fenotipos de acalasia comparten el elemento de la OTSUEG, pero el patrón de contractilidad esofágica varía desde contracciones espásticas por un lado y la ausencia de contractilidad por el otro.

Muchos estudios han confirmado que la respuesta al tratamiento va a depender del fenotipo del paciente, ya sea alguno de los tipos de acalasia o uno de los síndromes acalásicos (OTSUEG, espasmo esofágico distal, jackhammer, efecto opioide, obstrucción mecánica o contractilidad ausente).

Aunque no existen tratamientos curativos para los trastornos de la OTSUEG, existen opciones de tratamiento médico, dilatación neumática endoscópica (DN), miotomía endoscópica peroral (MEP), miotomía laparoscópica de Heller (MLH) y toxina botulínica; que en muchos casos estarán determinados por el fenotipo del paciente.

Varios estudios han observado una relación entre el tipo de OTSUEG (funcional vs. secundaria) y la opción terapéutica.

Uno de los primeros estudios de series de casos de OTSUEG describió 16 pacientes con OTSUEG funcional que fueron tratados con toxina botulínica, DN o MLH; los únicos que respondieron al tratamiento fueron tres pacientes llevados a miotomía en un tiempo de seguimiento de 12 meses $^{36}$.

La sintomatología del paciente puede guiar también el tratamiento a recibir, ya que en pacientes con sintomatología persistente, principalmente disfagia y sin evidencia de compromiso anatómico o infiltrativo que la explique, podría considerarse un tratamiento que genere disrupción del esfínter (por ejemplo, MEP, DN o MLH).

En pacientes con OTSUEG y sintomatología persistente distinta a disfagia, puede considerarse un tipo de tratamiento diferente. Hay que pensar que el diagnóstico manométrico simplemente pudo haber sido un hallazgo incidental o secundario a una anormalidad mecánica más que a una alteración neuromuscular. En estos casos no siempre hay que atribuírsele los síntomas a una falta de relajación del EEI. En el caso de que el síntoma sea reflujo, posiblemente la terapia a elegir no sea ninguna de las que altera el EEI. Muchos de estos pacientes presentan resolución espontánea de los síntomas con el seguimiento o reciben tratamiento con inhibidores de la bomba de protones con posterior resolución de los síntomas ${ }^{15,16}$.

En numerosos estudios se ha descrito que un gran porcentaje de los pacientes presentan durante el seguimiento una resolución de los síntomas sin necesidad de algún tratamiento específico. Pérez-Fernández, et al., en un estudio de pacientes con OTSUEG, reportaron que la mitad de los pacientes (52\%) no recibieron ningún tratamiento específico, de los cuales un $92 \%$ reportaron una resolución de 
los síntomas ${ }^{15}$. Es decir, más de un tercio de los pacientes con OTSUEG presentaron resolución espontánea de los síntomas. Resultados similares han sido descritos en otros estudios $^{10,16,21}$.

Un consenso de expertos fue publicado en el 2017 para el tratamiento de los trastornos motores esofágicos en la era de la MAR, enfocándose en describir los tratamientos más efectivos en los distintos síndromes acalásicos basándose en la información existente y en la opinión de expertos ${ }^{37}$. Para la OTSUEG reco-

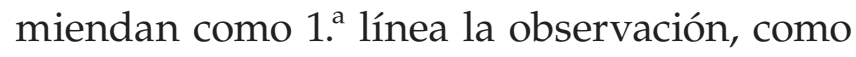
2. ${ }^{a}$ línea el uso de calcio antagonistas y como 3. a línea la aplicación de toxina botulínica. En caso de escoger algún tratamiento de los que clásicamente se utilizan para acalasia, recomiendan utilizar en primer lugar la $\mathrm{DN}$, seguido por el MEP.

Ihara, et al. ${ }^{38}$ describieron las estrategias de tratamiento para la OTSUEG en un centro de Japón. Ellos consideran razonable el uso de tratamiento médico no invasivo inicialmente, sabiendo que sus efectos terapéuticos son limitados. Sugieren que los pacientes que sean refractarios al tratamiento médico pueden ser llevados a tratamientos invasivos, ya que la OTSUEG puede ser una variante de la acalasia.

Se ha propuesto el uso de relajantes del músculo liso, como los nitratos y los bloqueadores de los canales de calcio ${ }^{39}$. Ambos actúan reduciendo la presión del EEI y la amplitud de contracción esofágica. El uso de estos medicamentos se ha descrito principalmente en la acalasia, en donde las dosis utilizadas son: para el nifedipino, 10-30 mg 30-45 minutos antes de las comidas, y para el dinitrato de isosorbida, $5 \mathrm{mg}$ 10-15 minutos antes de las comidas.

Los inhibidores de la fosfodiesterasa-5 (sildenafilo, 50-100 mg día) constituyen una nueva opción terapéutica, actuando mediante el bloqueo de la degradación del óxido nítrico, promoviendo su acción y generando una mayor relajación del músculo liso. Estos medicamentos reducen tanto la amplitud contráctil como la velocidad de propagación en pacientes con trastornos de la motilidad. La mejoría sintomática es muy variable con todos estos medicamentos, describiéndose en algunos estudios tasas de respuesta entre 0 y $50 \%$ en pacientes con OTSUEG $^{15,21}$; y los efectos adversos pueden ocurrir en un porcentaje considerable de pacientes (hasta en un 30\%). Además, la respuesta al tratamiento tiende a ser transitoria.

Se ha descrito el uso de acotiamida, que ejerce un efecto procinético y de mejoría en el vaciamiento gástrico, utilizada para el tratamiento de la dispepsia funcional. Actúa como un inhibidor de la colinesterasa. Un estudio describió que el $83.3 \%$ de pacientes con OTSUEG con síntomas tanto de ERGE como de dispepsia fueron tratados satisfactoriamente con este medicamento ${ }^{40}$. La puntuación del cuestionario de frecuencia para los síntomas de reflujo disminuyó con el uso de la acotiamida en el grupo de pacientes con OTSUEG de $19.0(6.0-38.0)$ antes del tratamiento a 9.5 (3.0-20.0) postratamiento.

La inyección de toxina botulínica en el EEI puede disminuir la presión inhibiendo las terminaciones colinérgicas excitatorias al bloquear la liberación de acetilcolina; sin embargo, la mayoría de los pacientes presentan recaídas en el primer año y los tratamientos 
repetidos tienen una menor eficacia a la terapia inicial. Sin embargo, estos tratamientos podrían ser una opción en pacientes que no sean candidatos a otras terapias más invasivas debido a comorbilidades más severas.

En otro estudio ${ }^{21}$, se realizó DN en nueve casos de posible OTSUEG secundaria a una estenosis o anillo en la UEG, reportando una respuesta sostenida (mejoría sintomática $>3$ meses) en solo el 12\%, transitoria en el 44\% y sin respuesta alguna en el $44 \%$. En este mismo estudio se realizó MEP en tres pacientes, todos cursando con disfagia como síntoma predominante sin explicación anatómica y que habían fallado a otro tipo de intervenciones previamente. Todos presentaron respuesta sintomática posprocedimiento.

Otro estudio llevó a tratamiento a 11 pacientes con OTSUEG secundaria, seis de ellos con estenosis (cuatro pépticas y dos esofagitis eosinofílicas) que fueron tratados con inhibidores de la bomba de protones y $\mathrm{DN}$, con mejoría de los síntomas a los 14 meses. Cuatro pacientes con hernia hiatal grande mejoraron con cambios dietarios, inhibidores de la bomba de protones y cirugía antirreflujo. Un paciente con divertículo epifrénico mejoró con diverticulotomía y cirugía antirreflujo ${ }^{11}$. Este mismo estudio mostró una respuesta al tratamiento durante un tiempo promedio de 8-12 meses, en 11 de 15 pacientes con OTSUEG funcional llevados a tratamiento con DN (cuatro pacientes) e inyección de botox (11 pacientes).

\section{CONCLUSIONES}

La OTSUEG constituye un grupo clínico heterogéneo que incluye pacientes con diversas causas, como alteraciones neuromusculares de la UEG (parecido a la acalasia), alteraciones mecánicas (por ejemplo, hernia hiatal), procesos infiltrativos (tumores, esofagitis eosinofílica), compresión extrínseca o simplemente un artefacto manométrico.

Por esto difícilmente puede haber un tratamiento estandarizado para esta condición y más bien debe ser individualizado, teniendo en cuenta factores como los síntomas del paciente y la severidad de estos, y el tipo de OTSUEG (funcional vs. secundaria), entre otras cosas.

Debido a que un porcentaje importante de pacientes presentan resolución espontánea de los síntomas con el seguimiento a unos meses, puede ser válido aplicar esta opción de tratamiento y definir de acuerdo a la evolución si llevar o no a una intervención terapéutica. El gran reto en el tratamiento de estos pacientes va a ser distinguir en qué momento y a qué pacientes hay que llevar a un tratamiento.

\section{BIBLIOGRAFÍA}

1. Bredenoord AJ, Weusten BL, Timmer R, Smout AJ. Intermittent spatial separation of diaphragm and lower esophageal sphincter favors acidic and weakly acidic reflux. Gastroenterology. 2006;130:334-40.

2. Kahrilas PJ, Bredenoord AJ, Fox M, Gyawali CP, Roman S, Smout AJPM, et al. The Chicago Classification of esophageal motility disorders, v3.0. Neurogastroenterol Motil. 2015;27:160-74.

3. Ghosh SK, Pandolfino JE, Rice J, Clarke JO, Kwiatek M, Kahrilas PJ. Impaired deglutitive EGJ relaxation in clinical esophageal manometry: a quantitative analysis of 400 patients and 75 controls. Am J Physiol Gastrointest Liver Physiol. 2007;293:G878-85.

4. Pandolfino JE, Kwiatek MA, Nealis T, Bulsiewicz W, Post J, Kahrilas PJ. Achalasia: A new clinically relevant classification by high-resolution manometry. Gastroenterology. 2008;135:1526-33.

5. Hani A, Bernal W, Leguízamo A, Zuluaga C, Vargas R, Vergara H, et al. Cómo realizar e interpretar una manometría esofágica de alta resolución usando la clasificación de Chicago 3.0. Rev Col Gastroenterol. 2017; 32:369-78.

6. Schlottmann F, Patti MG. Primary esophageal motility disorders: Beyond achalasia. Int J Mol Sci. 2017;18. 
7. Gyawali CP, de Bortoli N, Clarke J, Marinelli C, Tolone S, Roman S, et al. Indications and interpretation of esophageal function testing. Ann N Y Acad Sci. 2018 May 12. doi: 10.1111/nyas.13709. [Epub ahead of print]

8. Kahrilas PJ, Bredenoord AJ, Fox M, Gyawali CP, Roman S, Smout AJPM, et al. International Working Group for Disorders of Gastrointestinal Motility and Function. Advances in the management of oesophageal motility disorders in the era of high-resolution manometry: a focus on achalasia syndromes. Nat Rev Gastroenterol Hepatol. 2018;15:323.

9. Song BG, Min YW, Lee H, Min BH, Lee JH, Rhee PL, et al. Clinicomanometric factors associated with clinically relevant esophagogastric junction outflow obstruction from the Sandhill high-resolution manometry system. Neurogastroenterol Motil. 2018;30.

10. van Hoeij FB, Smout AJPM, Bredenoord AJ. Characterization of idiopathic esophagogastric junction outflow obstruction. Neurogastroenterol Motil. 2015;27:1310-6.

11. Clayton SB, Patel R, Richter JE. Functional and anatomic esophagogastic junction outflow obstruction: manometry, timed barium esophagram findings, and treatment outcomes. Clin Gastroenterol Hepatol. 2016;14:907-11.

12. DeLay K, Austin GL, Menard-Katcher P. Anatomic abnormalities are common potential explanations of manometric esophagogastric junction outflow obstruction. Neurogastroenterol Motil. 2016;28:1166-71.

13. Ravi K, Murray JA, Geno DM, Katzka DA. Achalasia and chronic opiate use: Innocent bystanders or associated conditions? Dis Esophagus. 2016;29:15-21.

14. Kraichely RE, Arora AS, Murray JA. Opiate-induced oesophageal dysmotility. Aliment Pharmacol Ther. 2010;31:601-6.

15. Pérez-Fernández MT, Santander C, Marinero A, Burgos-Santamaría D, Chavarría-Herbozo C. Characterization and follow-up of esophagogastric junction outflow obstruction detected by high resolution manometry. Neurogastroenterol Motil. 2016;28:116-26.

16. Lynch KL, Yang Y-X, Metz DC, Falk GW. Clinical presentation and disease course of patients with esophagogastric junction outflow obstruction. Dis Esophagus. 2017;30:1-6.

17. Roman S, Kahrilas PJ. Challenges in the swallowing mechanism: nonobstructive dysphagia in the era of high-resolution manometry and impedance. Gastroenterol Clin North Am. 2011;40:824-35.

18. Blonski W, Kumar A, Feldman J, Richter JE. Timed barium swallow: diagnostic role and predictive value in untreated achalasia, esophagogastric junction outflow obstruction, and non-achalasia dysphagia. Am J Gastroenterol. 2018;113:196-203.

19. Manuscript A, Magnitude S. A Comparison of symptom severity and bolus retention to chicago classification esophageal pressure topography metrics in patients with achalasia. 2013;31:1713-23.

20. Rohof WO, Lei A, Boeckxstaens GE. Esophageal stasis on a timed barium esophagogram predicts recurrent symptoms in patients with long-standing achalasia. Am J Gastroenterol. 2012;108:49-55.

21. Okeke FC, Raja S, Lynch KL, Dhalla S, Nandwani M, Stein EM, et al. What is the clinical significance of esophagogastric junction outflow obstruction? evaluation of 60 patients at a tertiary referral center. Neurogastroenterol Motil. 2017; 29:1-6

22. Bogte A, Bredenoord AJ, Oors J, Siersema PD, Smout AJ. Assessment of bolus transit with intraluminal impedance measurement in patients with esophageal motility disorders. Neurogastroenterol Motil. 2015;27:1446-52.

23. Tutuian R, Vela MF, Balaji NS, Wise JL, Murray JA, Peters JH, et al. Esophageal function testing with combined multichannel intraluminal impedance and manometry: Multicenter study in healthy volunteers. Clin Gastroenterol Hepatol. 2003;1:174-82.
24. Jain A, Baker JR, Rubenstein JH, Chen JW. Bolus clearance in esophagogastric junction outflow obstruction is associated with strength of peristalsis. Neurogastroenterol Motil. 2017;29.

25. Schupack D, Katzka DA, Geno DM, Ravi K. The clinical significance of esophagogastric junction outflow obstruction and hypercontractile esophagus in high resolution esophageal manometry. Neurogastroenterol Motil. 2017;29:1-9.

26. Roman S, Kahrilas PJ. Management of spastic disorders of the esophagus. Gastroenterol Clin North Am. 2013;42:27-43.

27. Gyawali CP, Kushnir VM. High-resolution manometric characteristics help differentiate types of distal esophageal obstruction in patients with peristalsis. Neurogastroenterol Motil. 2011;23.

28. Fontes LHS, Herbella FAM, Rodríguez TN, Trivino T, Farah JFM. Progression of diffuse esophageal spasm to achalasia: Incidence and predictive factors. Dis Esophagus. 2013;26:470-4.

29. Ang D, Misselwitz B, Hollenstein M, Knowles K, Wright J, Tucker E, et al Diagnostic yield of high-resolution manometry with a solid test meal for clinically relevant, symptomatic oesophageal motility disorders: serial diagnostic study. Lancet Gastroenterol Hepatol. 2017;2:654-61.

30. Xiao Y, Nicodème F, Kahrilas PJ, Roman S, Lin Z, Pandolfino JE. Optimizing the swallow protocol of clinical high-resolution esophageal manometry studies. Neurogastroenterol Motil. 2012;24:e489-96.

31. Mittal RK, Kassab G, Puckett JL, Liu J. Hypertrophy of the muscularis propria of the lower esophageal sphincter and the body of the esophagus in patients with primary motility disorders of the esophagus. Am J Gastroenterol. 2003;98:1705-12.

32. Krishnan K, Lin CY, Keswani R, Pandolfino JE, Kahrilas PJ, Komanduri S. Endoscopic ultrasound as an adjunctive evaluation in patients with esophageal motor disorders subtyped by high-resolution manometry. Neurogastroenterol Motil. 2014;26:1172-8.

33. Kwiatek MA, Pandolfino JE, Hirano I, Kahrilas PJ. Esophagogastric junction distensibility assessed with an endoscopic functional luminal imaging probe (EndoFLIP). Gastrointest Endosc. 2010;72:272-8.

34. Rohof WO, Hirsch DP, Kessing BF, Boeckxstaens GE. Efficacy of treatment for patients with achalasia depends on the distensibility of the esophagogastric junction. Gastroenterology. 2012;143:328-7.

35. Pandolfino JE, de Ruigh A, Nicodème F, Xiao Y, Boris L, Kahrilas PJ. Distensibility of the esophagogastric junction assessed with the functional lumen imaging probe (FLIP ${ }^{\mathrm{TM}}$ ) in achalasia patients. Neurogastroenterol Motil. 2013;25(6):496-501.

36. Scherer JR, Kwiatek MA, Soper NJ, Pandolfino JE, Kahrilas PJ. Functional esophagogastric junction obstruction with intact peristalsis: A heterogeneous syndrome sometimes akin to achalasia. J Gastrointest Surg. 2009;13:2219-25.

37. Kahrilas PJ, Bredenoord AJ, Fox M, Gyawali CP, Roman S, Smout AJPM, et al.; International Working Group for Disorders of Gastrointestinal Motility and Function. Advances in the management of oesophageal motility disorders in the era of high-resolution manometry: a focus on achalasia syndromes. Nat Rev Gastroenterol Hepatol. 2018;15:323.

38. Ihara E, Muta K, Fukaura K, Nakamura K. Diagnosis and treatment strategy of achalasia subtypes and esophagogastric junction outflow obstruction based on high-resolution manometry. Digestion. 2017;95:29-35.

39. Bassotti G, Annese V. Review article: pharmacological options in achalasia. Aliment Pharmacol Ther. 1999;13:1391-6.

40. Muta K, Ihara E, Fukaura K, Tsuchida O, Ochiai T, Nakamura K. Effects of acotiamide on the esophageal motility function in patients with esophageal motility disorders: a pilot study. Digestion. 2016;94:9-1. 